\title{
Ueber die Widerstandsfähigkeit von Klängen, insonderheit von Vocalklängen, gegenüber schädigenden Einflüssen.
}

\author{
Von
}

Max Fetzer, approb. Arzt aus Stuttgart.

(Mit 3 Textfiguren.)

E i n l e i t u n g.

Das tägliche Leben gibt zwar fortwährend die reichste Gelegenheit, Beobachtungen über die Verhältnisse der Fortpflanzung von Klängen und Geräuschen durch die verschiedenen Medien, insbesondere die atmosphärische Luft, und die hierbei auftretenden Erscheinungen zu machen. Und es lässt sich auch in der That bei einiger Aufmerksamkeit eine Reihe hierbei vorkommender interessanter Erscheinungen sicher beobachten und feststellen, von denen ich weiter unten zwei anführe. Aber trotz dieser vielfachen Beobachtungsgelegenheit einerseits ist doch wegen der grossen Schwierigkeit exacter Messungen andererseits dieses ganze Gebiet verhältnissmässig noch sehr wenig untersucht worden. Schon betreffs der fundamentalen Frage nach dem Verhältniss der Schallstärkeabnahme zur Entfernung von der Schallquelle kam noch in neuerer Zeit Vierordt' ${ }^{1}$, gestützt auf seine experimentellen Untersuchungen und ganz im Gegensatz zu den Forderungen der abstracten Theorie, zu dem Resultat, dass die Abnahme der Schallstärke in der freien Luft in einfachem Verhältniss zur Entfernung von der Schallquelle stehe.

- Ohne auf diese von anderer Seite - wohl mit Recht angefochtene Behauptung ${ }^{2}$ ) näher einzugehen, soll in Folgenden

1) C. v. Vierordt, Die Schall- und Tonstärke und das Schallleitungsvermögen der Körper S. 245. Tübingen 1885.

2) M. Wien, Ueber die Messung der Tonstärke, Wiedemann's Annalen Bd. 36 S. 834, 1889 und derselbe, Ueber die Empfindlichkeit des menschlichen Ohres u. s. w. Pflüger's Archiv Bd. 97 S. 1, 1908, sowie F. H. Quix, Over de intensiteit van het geluid van eene stemvork, zoals die afhankelijk is van 
vielmehr eine Reihe von Untersuchungen und Beobachtungen angeführt werden, wie sie sich durch ein musikalisch geübtes Ohr, allein oder durch einfache Apparate unterstützt, anstellen liessen. Die hierbei festgestellten Ergebuisse können ihrer Subjectivität wegen natürlich keine absoluten Werthe beanspruchen.

Indem ich, wie oben erwähnt, von einigen allgemein bekannten Erscheinungen des täglichen Lebens ausgehe, führe ich zunächst folgende Beobachtung an. Wenn man sich im Freien einer irgendwo spielenden Musikcapelle, die eine Summe der verschiedensten Tonhöhen, Tonstärken und Klangfarben repräsentirt, nähert, so kann man vielfach beobachten, dass in grösserer Entfernung zunächst nur die tiefen, meist begleitenden Basstöne gehört werden können, während die Wahrnehmung der höher klingenden, meist melodieführenden Stimmen erst nach weiterer Annäherung hinzutritt. Umgekehrt hat diese Thatsache C. M. v. Weber im ersten Acte des "Freischütz" in sehr feiner und glücklicher Weise benutzt, um im Hörer die Illusion einer abziehenden Bauernmusik zu erwecken, indem er das Orchester, welches die abziehende Bauermusik wiedergibt, nicht bloss immer leiser, immer mehr piano spielen, sondern gerade die melodieführenden höheren Stimmen allmählich ausfallen lässt und nur noch den Bass beibehält. Diese an sich leicht zu erklärende Erscheinung - erzeugen ja doch die tiefen Instrumente des Orchesters meist auch die grösste Tonintensität - ist doch insofern interessant, als der Hörer von demselben Orchester, von dem er in beträchtlicher Entfernung nur die tiefsten Töne (in Folge ihrer grössten Tonintensität) wahrzunehmen im Stande war, in der Nähe keineswegs den Eindruck bekommt, als seien die erwähnten tiefen Töne besonders laut. Und zwar ist dies nicht nur beim Laien der Fall, der daran gewöhnt ist, vor Allem oder ausschliesslich die melodieführenden, zumeist also die höheren Stimmen zu beachten, ind nicht geschult ist, auch die Mittel-, Füllund begleitenden Stimmen mit Aufmerksamkeit zu verfolgen, (dessen Wahrnehmungsvermögen für die tieferen Töne also in Folge mangelnden oder geringeren Vermögens, von den höheren zu abstrahiren, vielleicht vermindert ist), sondern es gilt dies auch vom Musiker, der im Stande ist, einer begleitenden Stimme seine con-

amplitudo en afstand. Onderzoekingen, gedaan in het Physiologisch Laboratorium der Utrechtsche hongeschool. vijfve reeks. Deel 3 p. 240. 1901, woselbst die weitere Literatur zu finden ist. 
centrirte Aufmerksamkeit zuzuwenden. Ja der Letztere wird im Gegentheil, besonders, wenn er es mit geringerev Blasorchestern zu thun hat, die hierbei gern verwendeten höheren Clarinettentöne als besonders laut und schreiend empfinden, dieselben Töne, die er in der Ferne kaum wahrnehmen konnte.

Auf diese Erscheinungen, auf die ich unten wieder zurückkomme, weisen auch $\mathrm{H}$. $\mathrm{Z}$ wa ardemaker und F. H. Quix ${ }^{1}$ ) hin, wenn sie schreiben: „De luidheid en de physische intensiteit van een toon zijn twee verschillende dingen; het eerste is de sterkte van den indruk, die ons gehoororgaan verkrijgt, het tweede is het betrekkelijk quantum acustische energie, in natuurkundigem zin, dat tot het orgaan doordringt. Op het gevoeligste punt der toonladder, als het noare de gele vlek onzer gehoorlijn, kan dit quantum hoogst onbeduidend en de indruk toch hijrouver levendig zijn; in de minder gevoelige zonen kan zulk een energie-quantum zeer groot worden en desniettemin onopgemerkt blijven."

Noch auffälliger treten diese Verbältnisse bei den menschlichen Sprachlauten in die Erscheinung. Von einer im Freien gehaltenen Rede oder gesprochenen Unterhaltung, die für den Nahestehenden den Eindruck ziemlich gleichmässiger Stärke macht, hören wir in einiger Entfernung nur mehr einige Laute. Ganz hesonders merkwürdig ist ja dies, wie auch Helmholtz erwähnt ${ }^{2}$ ), in höherer Luft, wenn Luftschiffer oder Bergsteiger Gelegenheit haben, Gespräche unter ihnen befindlicher Personen mit anzuhören; auch hierbei dringen an das Ohr des höher stehenden Beobachters nur ganz vereinzelte Sprachlaute. Dass es solche gibt, die ganz besonders weit zu dringen vermögen, ist andererseits allgemein bekannt. Es sind dies z. B. besonders die Laute $a$ und $o$. Dieser Vocale wird sich im Wesent-

1) H. Zwaardemaker en F. H. Quix, Onze gehoorscherpte voor de tonen van middenoctaven en discant. Nederl. Tijdscbrift voor geneeskunde. Deel 2 Nr. 9 p. 3. 1902.

2) H. Helmboitz, Die Lehre von den Tonempfindungen als physiol. Grundlage für die Theorie der Musik S. 118: „Bei recht stillem Wetter ist es interessant, von hohen Betgen herab die Stimmen der Menschen aus der Ebene zu belauschen. Worte sind danı nicht mehr erkennbar oder höchstens solche, welche aus $M, N$ und blossen Vocalen zusammengesetzt sind, wie Mama, nein. Aber die in den gesprochenen Worten enthaltenen Vocale unterscheidet man leicht und deutlich. Sie folgen sich in seltsamem Wechsel und wunderlich erscheinenden Tonfällen, weil man sie nicht mehr zu Worten und Sätzen zu verbinden weiss. 
lichen und in passender Verbindung auch Der bedienen, der sich einer weit entfernten Person bemerkbar machen will (hallob, hollah u. s. w.).

Einen Versuch, die Tonstärkeverhältnisse der einzelnen Sprachlaute zu einander experimentell festzustellen, fand ich bei $\mathrm{Wolf}^{1}$ ). Die unten angeführten Ergebnisse stellen natülich keine allgemein geltenden Entfernungen dar, da ja alle in Betracht kommenden Verhältnisse (Stimme des Sprechers, Gehör des Beobachters, Besehaffenheit der äusseren Luft $u$. s. w.) bei den verschiedenen Untersuchungen stets verschieden sind, sondern nur das Verhältniss der Tonstärke der einzelnen Sprachlaute zu einander, im Speciellen der Vocale. Wolf ging so vor, dass er bei windstillem Wetter auf einer zu beiden Seiten mit hohen Bäumen dicht bepflanzten Chaussée, die also eine Art Lautgang bildete und zu beiden Seiten von Wald begrenzt war, von einem Freunde die einzelnen Vocale in möglichst gleichmässig fortdauernder Tonstärke angeben liess, während er selbst, rückwärts gehend, die Entfernung feststellte, in welcher der betreffende Vocal nicht mehr deutlich unterschieden werden konnte. Folgende Tabelle gibt die wesentlichen Resultate der Versuche Wolf's (hier nur in Betreff der Vocale).

Schematische Darstellung des Tonstärkeverhältnisses der Sprachlaute (nach 0 . Wolf).

\begin{tabular}{l|c|c}
\hline $\begin{array}{c}\text { Sprach- } \\
\text { laut }\end{array}$ & $\begin{array}{c}\text { Wird noch deutlich } \\
\text { unterschieden in einer } \\
\text { Entfernung von Schritten }\end{array}$ & B e m e r k u n g e n \\
\hline$A$ & 360 & *) Unter oben angefü hrten Verhältnissen \\
und geschilderter Methode gemessen \\
$O$ & 350 & **) Klingt meist wie $\boldsymbol{A i}$ \\
$\left.E i^{* *}\right)$ & 340 & \\
$E$ & 330 & \\
$I$ & 300 & \\
$E u$ & 290 &
\end{tabular}

Am weitesten und besten wird also von Vocalen unter sonst gleichen Beringungen vernommen das $A$; ihm folgt das $O$, dann das $E$ und $I$. Und an wenigsten weit wird das $U$ gehört.

Schliesslich haben, soviel ich weiss, nur noch Grützner und Sauberschwarz ${ }^{2}$ ) Versuche angestellt, die mit den obigen eine

1) Dr. O. Wolf, Sprache und Ohr S. $58 \mathrm{ff}$.

2) E. Sauberschwarz, Interferenzversuche mit Vocalklängen. Pflüger's Arcb. Bd. 61 S. 1.1895. 
gewisse, wenn auch nur schwache Aehnlichkeit haben. Es wurden durch zweckmässige Apparate aus den verschiedenen Vocalklängen auf dem Wege der Interferenz entweder der Grundton oder bestimmte Obertöne ausgelöscht und die Aenderung des Vocalklanges untersucht. Im Allgemeinen ergab sich - was für uns von Interesse ist -, dass, wenn man den Grundton eines Vocales (und seine ungeradzahligen Obertöne) beseitigt, die Erkennbarkeit eines Vocales um so mehr beeinträchtigt wird, je höher er gesungen wird. Ferner gibt es gewisse individuelle Verschiedenheiten, indem die verschiedenen Vocale hierdurch in sehr verschiedener Weise geschädigt werden. "Am wenigsten beeinträchtigt wird wieder das $A$, am meisten das $U$; die übrigen Vocale stehen in der Mitte."

Vernichtete man weiter die charakteristischen Obertöne oder die Formanten, so wurden die Vocalklänge durchweg stärker beeinflusst. Während sie im ersten Fall oft nur wie aus weiter Ferne klangen, werden sie jetzt vielfach wie ein tiefes, unbestimmtes Brummen gehört. Nur das $A$ und einigermaassen auch das $O$ zeigen Ausnahmen, indem namentlich das erstere noch als solches zu erkennen ist.

Auch Hermann ${ }^{1}$ ) hat bei der mikrophonischen und telephonischen Uebertragung von Vocalen Aehnliches beobachtet und äussert sich hierüber folgendermaassen: "Am sichersten unter allen Vocalen erkennbar ist $A$. Die Vocale $E, I, A e, O e, U e$ werden sehr leicht mit einander verwechselt, dagegen fast niemals mit $A, O$ oder $U$. $O$ wird mit $U$ leicht verwechselt, fast nie einer dieser Vocale mit $A . "$

Da mir nun Herr Prof. v. Grützner mittheilte, dass er schon früher verschiedene Versuche, ähnlich denen von Wolf, gemacht hatte - auf welche ich noch später zurückkommen werde - , und dass diese Versuche beachtenswerthe Ergebnisse geliefert hätten, so ging ich um so lieber auf seinen Vorschlag ein, die Angelegenheit weiter experimentell zu prüfen, als sie mich als langjährigen Violinspieler, der über ein gutes musikalisches Gehör verfügt, ganz besonders interessirte.

1) L. Hermann, Die Uebertragung der Vocale durch das Telephon und das Mikrophon. Pflüger's Arch. Bd. 48 S. 543. 1891. 


\section{Eigene Versuche.}

\section{A. Abschwächung von Vocalklängen durch die freie Luft.}

Ich machte mich nun daran, zunächst die Versuche von Wolf zu wiederholen und zu prüfen, ob auch ich unter ähnlichen, wenn auch nicht vollkommen gleichen Bedingungen ähnliche Ergebnisse erhalten würde. Es wurde also festgestellt, in welcher Entfernung der auf einen bestimmten Ton gesungene Vocal überhaupt noch gehört wird, und ferner, wie das betreffende Fortpflanzungsmedium, im vorliegenden Falle also die atmosphärische Luft, im Stande ist, den Charakter des Vocals anzugreifen und zu verändern.

Die Versuche wurden auf der ebenen Landstrasse LustnauBebenhausen, die in Länge von mehreren Kilometern sich in gerader Richtung erstreckt, angestellt. Die jeweiligen Entfernungen zwischen dem Rufenden und dem Hörer konnten nach den staatlichen Längenmarkirungen der Strasse stets genau festgelegt werden. Parallel der Landstrasse verliefen beiderseits in etwa 200-400 m Entfernung mässig hohe, bewaldete Höhenzüge. Zwischen diesen und der Strasse lagen ebene Wiesen, die nur mit wenigen, kleinen Obstbäumen bepflanzt waren. Gebäulichkeiten waren in der Umgebung nicht vorhanden. Die unten mitgetheilten Versuche wurden nach einem Regentag an einem hellen, fast windstillen Vormittag im August 1902 vorgenommen. Der ganz leichte Wind, der sich zeitweise erhob, schnitt die Strasse, somit auch die in Betracht kommende Schallrichtung, in ungefähr rechtem Winkel. Die äusseren Verbältnisse liessen somit eine ziemlich genaue Untersuchung zu.

Um auch bei den Experimentirenden, Rufer wie Hörer, Fehlerquellen möglichst zu vermeiden, hatten Beide je einen Assistenten zur Seite. Bei Ersterem handelte es sich vor Allem um ein in gleicher Tonstärke, Tondauer und Tonhöhe gehaltenes Rufen der betreffenden Vocale, was der bejgegebene, musikalisch geübte Assistent zu controliren hatte, etwaige Abweichungen protokollirend; bei Letzterem sollten durch das vergleichende Urtheil des Assistenten Fehlerquellen, wie sie durch die wechselnde Intensität der Aufmerksamkeit des Hörers hervorgerufen werden, möglichst beseitigt werden.

Gerufen wurden die Vocale natürlich so deutlich und rein wie möglich mit den charakteristischen Mundstellungen, wie sie zur Bildung des betreffenden Vocals nöthig sind, in einer dem Rufenden möglichst 
bequemen, aber unveränderlich festgehaltenen Tonhöhe, was durch kleine Pfeifen controlirt wurde, und, wie oben schon erwähnt, in immer gleicher Dauer und (subjectiver) Stärke. Jeder Vocal wurde dem Hörer vorher durch ein optisches Signal angekündigt, was besonders für grössere Entfernungen, bei denen einzelne Vocale für den Hörer ganz ausfielen, im Interesse einer sicheren Beobachtung nöthig war. Von der Verwendung von Sprachrohren oder Schallbechern, auch solchen von den Händen des Rufers gebildeten, wurde abgesehen.

Im Folgenden gebe ich zunächst tabellarisch die Resultate dieser Versuche wieder, wobei ich bemerke, dass die Tabellen aus den Ergebnissen zusammengestellt sind, die sich durch mehrmalige Controlversuche, die ja schon durch die im Freien immer wieder eintretenden Störungen, wie in Folge von Windstörungen oder Geräuschen u.s. w., nöthig wurden, immer wieder sicher feststellen liessen.

\begin{tabular}{|c|c|c|c|c|c|}
\hline Tonhöhe & Tonstärke & $\begin{array}{c}\text { Entf. } \\
\text { m }\end{array}$ & $\begin{array}{l}\text { Vocal } \\
\text { ges. von } \\
\text { M. F. }\end{array}$ & $\begin{array}{c}\text { Gehört } \\
\text { von } \\
\text { H. G. }\end{array}$ & Bemerkungen \\
\hline$c i s^{\prime}\left(\frac{1}{2}=\right.$ & 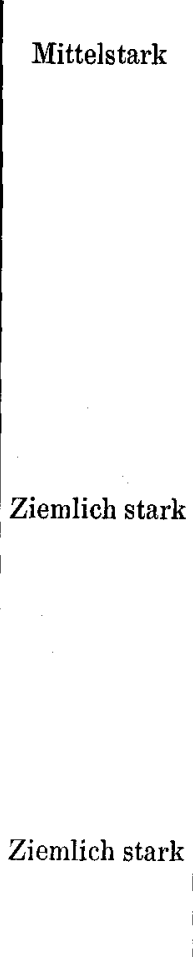 & 125 & $\begin{array}{l}A \\
I \\
E \\
U \\
\\
O \\
U \\
I \\
E \\
A \\
O \\
U \\
I \\
A \\
E \\
O \\
A \\
E \\
I \\
O \\
U \\
E \\
I \\
U \\
O \\
E \\
A \\
I \\
A \\
E \\
I \\
A\end{array}$ & $\begin{array}{l}A \\
I \\
E \\
U \\
O \\
U \\
I \\
E \\
A \\
O \\
U \\
I \\
A \\
E \\
O \\
A \\
E \\
I \\
O \\
U \\
E \\
I \\
U \\
O \\
E \\
A \\
I \\
A \\
E \\
I \\
A\end{array}$ & $\begin{array}{l}\text { Etwas schwächer als die } \\
\text { vorhergehenden } \\
\text { Etwas schwächer } \\
\text { Stark } \\
\text { Ziemlich schwach } \\
\text { Sehr detitlich } \\
\text { Schwach } \\
\text { Schwächer als } E \\
\text { Schwächer als } E \\
\text { Sehr stark } \\
\text { Schwach } \\
\text { Stark }\end{array}$ \\
\hline
\end{tabular}




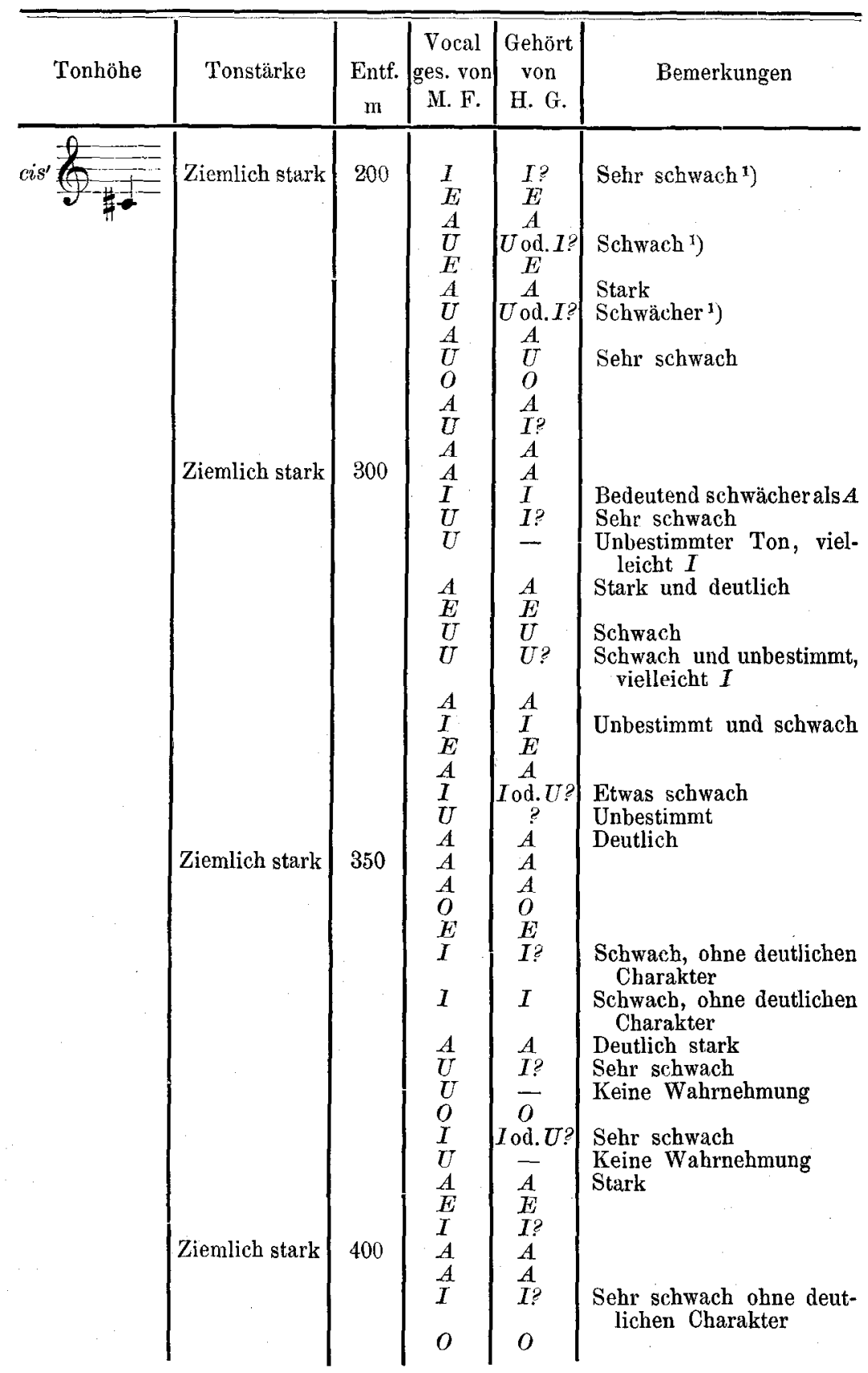

1) Das Fragezeichen hinter dem Vocal bedeutet, dass der Hörer nicht ganz sicher war, den betreffenden Laut zu vernehmen. 


\begin{tabular}{|c|c|c|c|c|c|}
\hline Tonhöhe & Tonstärke & $\begin{array}{c}\text { Entf. } \\
\text { m }\end{array}$ & $\begin{array}{c}\text { Vocal } \\
\text { ges. von } \\
\text { M. F. }\end{array}$ & $\begin{array}{c}\text { Gehört } \\
\text { von } \\
\text { H. G. }\end{array}$ & Bemerkungen \\
\hline $\operatorname{cis}^{\prime}\left(\frac{1}{2}=\right.$ & $\begin{array}{l}\text { Ziemlich stark } \\
\text { Ziemlich stark } \\
\\
\end{array}$ & 650 & $\begin{array}{l}U \\
U \\
I \\
E \\
A \\
O \\
E \\
I \\
U \\
A \\
E \\
A \\
O \\
U \\
I \\
A \\
U \\
A \\
I \\
\\
E \\
O \\
U \\
A \\
O \\
I \\
A \\
A \\
O \\
U \\
U \\
I \\
\\
U \\
O \\
A \\
E \\
I \\
U \\
U \\
A \\
A \\
E \\
\\
A \\
A \\
I \\
U \\
U \\
I \\
O \\
E \\
I \\
I \\
A \\
U \\
O\end{array}$ & 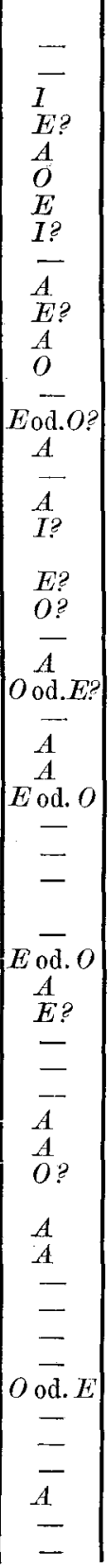 & $\begin{array}{l}\text { Keine Wabrnehmung } \\
\text { Keine Wahrnehmung } \\
\text { Sehr schwach } \\
\text { Zweifelhaft } \\
\text { Deutlich } \\
\text { Schwach u. zweifelhaft } \\
\text { Keine Wahrnehmung } \\
\text { Stark } \\
\text { Zweifelhaft } \\
\text { Schwächer als } A \\
\text { Keine Wahrnehmung } \\
\text { Schwach } \\
\text { Keine Wahrnehmung } \\
\text { Deutlich } \\
\text { Sehr schwach, kein Vocal- } \\
\text { charakter } \\
\text { Ziemlich schwach } \\
\text { Etwas starker } \\
\text { Keine Wahrnehmung } \\
\text { Schwach } \\
\text { Keine Wahrnehmung } \\
\text { Deutlich } \\
\text { Ziemlich stark } \\
\text { Keine Wahrnehmung } \\
\text { Keine Wahrnehmung } \\
\text { Schwacher, unbest. Ton, } \\
\text { vielleicht } U \\
\text { Keine Wahrnehmung } \\
\text { Unbestimmt } \\
\text { Unbestimmt, sehr schwach } \\
\text { Keine Wahrnehmung } \\
\text { Keine Wahrnehmung } \\
\text { Keine Wahrnehmung } \\
\text { Unbest., sehr schwacher } \\
\text { Ton } \\
\text { Deutlich } \\
\text { Keine Wahrnehmung } \\
\text { Keine Wahrnehmung } \\
\text { Keine Wahrnehmung } \\
\text { Ganz schwach, vielleicht } U \\
\text { Ziemlich schwach } \\
\text { Sehr schwach, unbestimmt } \\
\text { Keine Wahrnehmung } \\
\text { Keine Wahrnehmung } \\
\text { Deutlich } \\
\text { Keine Wahrnehmung } \\
\text { Schwacher, unbest. Ton }\end{array}$ \\
\hline
\end{tabular}




\begin{tabular}{|c|c|c|c|c|c|}
\hline Tonhöhe & Tonstärke & $\begin{array}{c}\text { Entf. } \\
\text { m }\end{array}$ & $\begin{array}{l}\text { Vocal } \\
\text { ges. von } \\
\text { M. F. }\end{array}$ & $\begin{array}{c}\text { Gehört } \\
\text { von } \\
\text { H. G. }\end{array}$ & Bemerkungen \\
\hline $\operatorname{cis}^{\prime} \frac{}{\left(\frac{1}{2}-1\right.}$ & $\begin{array}{l}\text { Ziemlich stark } \\
\text { Ziemlich stark }\end{array}$ & $\begin{array}{l}650 \\
700\end{array}$ & $\begin{array}{l}E \\
A \\
A \\
A \\
U \\
U \\
I \\
E \\
A \\
O \\
O \\
U \\
I \\
U \\
E \\
A \\
A \\
O\end{array}$ & $\begin{array}{l}\bar{A} \\
A \\
\bar{A} \\
\overline{-} \\
\vec{A} \\
\bar{E} \\
\overline{-} \\
\overline{-} \\
\vec{A} \\
\bar{A}\end{array}$ & $\begin{array}{l}\text { Keine Wahrnehmung } \\
\text { Deutlich } \\
\text { Deutlich } \\
\text { Keine Wahrnehmung } \\
\text { Keine Wahrnehmung } \\
\text { Keine Wahrnehmung } \\
\text { Keine Wahrnehmung } \\
\text { Schwacher, unbest. Ton } \\
\text { Sehr schwach } \\
\text { Keine Wahrnehmung } \\
\text { Keine Wahrnehmung } \\
\text { Keine Wahrnehmung } \\
\\
\text { Schwacher. Ton }\end{array}$ \\
\hline
\end{tabular}

Die Resultate unserer Versuche sind also in Uebereinstimmung mit Wolf etwa folgende: Von den gleich laut gesungenen Vocalen besitzt die grösste Widerstandsfähigkeit das $A$; dann folgt das $O$, weiter $\boldsymbol{E}, I$ und zuletzt $U$. Aber schon bei Entfernungen, bei denen die subjectiven Tonstärken der gesungenen Vocale noch lange nicht bis zum Schwellenwerth herabgedrückt waren, bei denen man also noch einen Klang hörte, sind die den betreffenden Vocalcharakter bedingenden Momente, also die in dem Vocalklang enthaltenen Töne von bestimmter Höbe, seien es nun durch Resonanz verstärlite harmonische Obertöne [Helmholtz'), Pipping ${ }^{2}$, Hensen ${ }^{3}$ )] oder selbstständige Mundtöne harmoniseher oder unharmonischer Natur [Hermann ${ }^{4}$ )], merklich zerstört oder aufgehoben. Eine alleinige Ausnahme macht das $A$. Dieses wurde auch auf weiteste

1) H. Helmholtz, Lehre von den Tonempfindungen. 3. Ausgabe 1870 S. 162 ff.

2) H. P ipping, Zur Klangfarbe der gesungenen Vocale. Zeitschr. f. Biol. Bd. 27 N. F. Bd. 9 S. 77. 1890.

3) V. Hensen, Ueber die Schrift von Schallbewegungen. Zeitschr. f. Biol. Bd. 23 S. 291. 1887. - Die Harmonie in den Vocalen. Ebenda Bd. 28 S. 39. 1891.

4) L. Hermann, Phonographische Untersuchungen. Pflüger's Archiv Bd. 45 S. 582. 1889. Bd. 47 S. $42,44,347.1890$. Bd. 48 S. 543 u. 574.1891. Bd. 49 S. $499.1891 . \quad$ Bd. 53 S. 1. $1892 . \quad$ Bd. 56 S. $467.1894 . \quad$ Bd. 58 S. 255 u. 264. 1894. Bd. 61 S. 169 . 1895. Bd. 83 S. 1. 1900. - Annalen der Physik und Chemie: N. F. Bd. 58 S. 391. 1896. 
Entfernungen, wenn es überhaupt noch gehört werden konnte, stets richtig als $A$ erkannt. Es ist so zu sagen nicht umzubringen. Dagegen verloren schon auf $200 \mathrm{~m}$ Entfernung das $U$, etwas später auch das $I$ so sehr ihren Vocalcharakter, dass beide nur als schwacher, dumpfer 'Ton gehört und in Folge dessen häufig mit einander verwechselt wurden. Auf etwa $400 \mathrm{~m}$ Entfernung wurde auch das $E$ zweifelhaft, wogegen das $O$ immer noch deutlich erkannt werden konnte. Letzteres wurde auf grössere Entfernungen (etwa von $550 \mathrm{~m}$ ab) so verändert, dass es theils nur als hohler Ton, vergleichbar dem einer angeblasenen Orgelpfeife, theils merkwürdiger Weise häufig als $E$ oder $O e$ gehört wurde.

Aehnliche Versuche hatte ich, wie früher schon Grützner (siehe oben), Gelegenheit im Gebirge an einem sehr deutlichen Echo anzustellen. Die Versuchsanordnung war äbnlich der der oben erwähnten Experimente auf der Landstrasse. Rufer und Hörer waren natürlich dieselbe Person. Der vom Schall zurückgelegte Weg wurde aus der Zeitdifferenz zwischen Ruf und Echo bestimmt. Diese Fntfernung blieb unverändert. Die Resultate waren übereinstimmend mit den oben erwähnten. Auch bier konnten die Vocale nach ihren Lautheiten geordnet werden in die Reihe $A, O, E, I, U$. Verwechslungen der Vocale unter sich waren natürlich nicht möglich, da Rufer und Hörer eine Person waren; dagegen konnte deutlich beobachtet werden, dass bei entsprechend veränderten Tonstärken $U$ und $I$ als dumpfe, $O$ und $E$ als mehr hohle (orgelpfeifenartige) Töne ohne deutlichen Vocalcharakter zurückkamen, wogegen $A$ niemals beeinfusst, sondern stets als deutliches und klares $A$ gehört wurde.

B. Abschwächung von Vocalklängen durch feste Gegenstände und Luft.

War in den erstbeschriebenen Versuchen für den gesungenen Vocalklang nur der. Widerstand der atmospbärischen Luft zu überwinden, und trat dazu im Jetztbeschriebenen noch die Reflexion einer festen Wand, so sollte im Folgenden noch die Wirkung auf den Vocalklang von Luftwiderstand und Reflexion, verbunden mit dem Widerstand verschieden dichter Medien untersucht werden. Diese, sowie die später zu beschreibenden Versuche stellte ich im physiologischen Institut zu Tübingen im Sommersemester 1902 und im Winter- 
semester 1902/03 an. Auch im Sommersemester 1903 machte ich noch einige Controlversuche.

Fis wurden zu diesem Zweck die drei in einer Flucht liegenden, durch zwei $20 \mathrm{~cm}$ dicke gemanerte Wände, sowie bei $a$ und $b$ (siehe Fig. 1) durch die geschlossenen, je auf beiden Seiten mit dickem

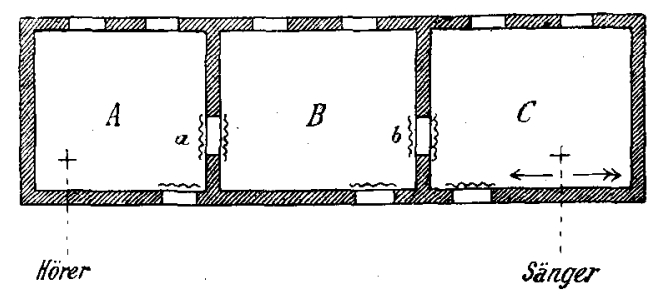

Fig. 1.

Wollstoff verhängten Holzthüren gesehiedenen Zimmer des Instituts $A, B$ und $C$ derart benutzt, dass in $C$ gesungen, in $A$ beobachtet wurde.

Die Höhe der drei Zimmer beträgt $4 \mathrm{~m} 20 \mathrm{~cm}$, ihre Breite $5 \mathrm{~m} 50 \mathrm{~cm}$, die Länge des Zimmers $A 6 \mathrm{~m}$, der Zimmer $B$ und $C$ je $7 \mathrm{~m}$. Auf der einen Längsseite besitzt jedes Zimmer, wie Fig. 1 zeigt, zwei grosse Fenster, während sich auf der gegenüberliegenden Seite je eine, während der Versuche natürlich geschlossene und mit dicken Teppichen verhängte, auf einen gemeinsamen Corridor führende Thüre befindet. Die an den Breitseiten befindlichen Verbindungsthüren sind oben erwähnt.

Sang der Sänger in der Richtung des Pfeiles $\leftarrow$, so konnten in $A$ noch alle Vocale deutlich erkannt werden, jedoch in so verschiedener Intensität, dass das $U$ sehr leise wahrgenommen wurde, während das $A$ stets laut und deutlich gehört wurde. Zwischen diesen Extremen standen wieder, wie bei den bisherigen Versuchen, nach zunehmender Lautheit geordnet, $I, E$ und $O$.

Nun wurde in $C$ in der Richtung des Pfeiles $\rightarrow$ gesungen. Das Ergebniss war wieder: Abnehmende Lautheit von $A$ nach $U$ über $O, E$ und $I$. $U$ und $I$ wurden theils als schwache, dumpf-summende Töne ohne bestimmten Vocalcharakter wahrgenommen, theils mit einander verwechselt. $E$ wird theils erkannt, theils als $I$ oder $U$ gedeutet; $O$ und $A$ wird deutlich gehört. (Vergl. Tabelle.) 
Versuch.

Vocale gesungen auf e $\underline{\underline{9 \div \bar{E}} \bar{E}}$

\begin{tabular}{c|l}
\hline \hline $\begin{array}{c}\text { Gesungen } \\
\text { von N. L. }\end{array}$ & \multicolumn{1}{|c}{ Gehört von M. F. } \\
\hline$A$ & A laut und deutlich \\
$I$ & Unbestimmter Ton \\
$O$ & $O$ \\
$U$ & Vielleicht $I$ \\
$U$ & Ganz schwach summender Ton \\
$A$ & $A$ \\
$O$ & $O$ \\
$E$ & Unbestimmt $I$ ? oder $E ?$ \\
$U$ & $I ?$ \\
$A$ & $A$ \\
$E$ & $E$ \\
$A$ & $A$ \\
$E$ & Unbestimmt $I$ oder $U$ \\
$U$ & Schwach $U$
\end{tabular}

C. Abschwächung von Klängen durch poröse Medien und durch Luft.

Im vorigen Versuch stellten den Hauptwiderstand für die Fortpflanzung des Tones die beiden gemauerten $Z$ wischenwände mit ihren verhängten Thüren, also ziemlich dichte, wenig poröse Materialien dar. Nun hat in letzter Zeit $\mathrm{Tufts}^{1}$ ) Untersuchungen über die Fortpflanzung von Luftströmungen und Tönen durch poröse Materialien angestellt. Als Widerstände verwendete er gleich dicke Glasröhren, welche in kürzeren oder längeren Strecken mit Schrot gefullt waren. Die Durchmesser der drei benutzten Schrotsorten betrugen 4,37, 2,79 und 1,22 mm. Bei continuirlichen Luftströmungen, welche Tufts zuerst untersuchte, konnte die Differenz der Drücke vor und nach Passirung der Widerstände direct durch ein Manometer abgelesen werden; bei den Tönen konnten die verschiedenen Intensitäten, ohne oder nach Einschaltung der Widerstände, aus den durch ein Fernrohr beobachteten verschiedenen Amplituden einer durch die Tonquellen in Schwingung versetzten Membran erkannt werden. Als Tonquelle diente eine stets in gleicher Stärke angeblasene Orgelpfeife. Betreffs der näheren Einzelheiten der Versuchsanordnung darf ich wohl auf den oben angeführten Originalaufsatz

1) F. L. Tufts, The transmission of Sound trough porous materials. American Journal of science Vol. 11 Art. 29 S. 357. 1901. 
selbst verweisen. Tufts kam durch seine Versuche zu folgenden Resultaten: Für Luftströmungen sowohl wie für die Fortpflanzung von Tönen ist der Widerstand körniger Materialien bei gleichem Korn proportional der Schichtböhe, bei verschiedenem Korn diesem etwa umgekehrt proportional (oder, anders ausgedrückt, proportional der Dichtigkeit des Widerstandes bei sonst gleichen Verhältnissen). Letzterer Satz hat jedoch nicht immer Gültigkeit; vielmehr zeigen sich Verschiedenheiten, besonders bei kleinkalibrigen körnigen Materialien, je nachdem sich der Widerstand in der Nähe eines Schwingungsknotens oder eines Schwingungsbauches befindet. Für meine unten näher beschriebene subjective Methode wurde nun der Vorgang von Tufts insoweit benutzt, als auch hierbei als zu passirende Widerstände für die Töne und Vocale körnige Materialien verwendet wurden, die, zum Theil von gleichem, bekanntem Kaliber, wie Schrot, oder von unregelmässigem, wie grobgepulvertes Glas u. s. w., in Säulen von gleichem Durchmesser und verschiedenen Längen aufgeschichtet wurden.

$\mathrm{Zu}$ diesem Zweck wurden abgemessene Volumina der betreffenden Materialien in ein U-förmiges Glasrohr $A B$ (s. Fig. 2) eingefüllt,

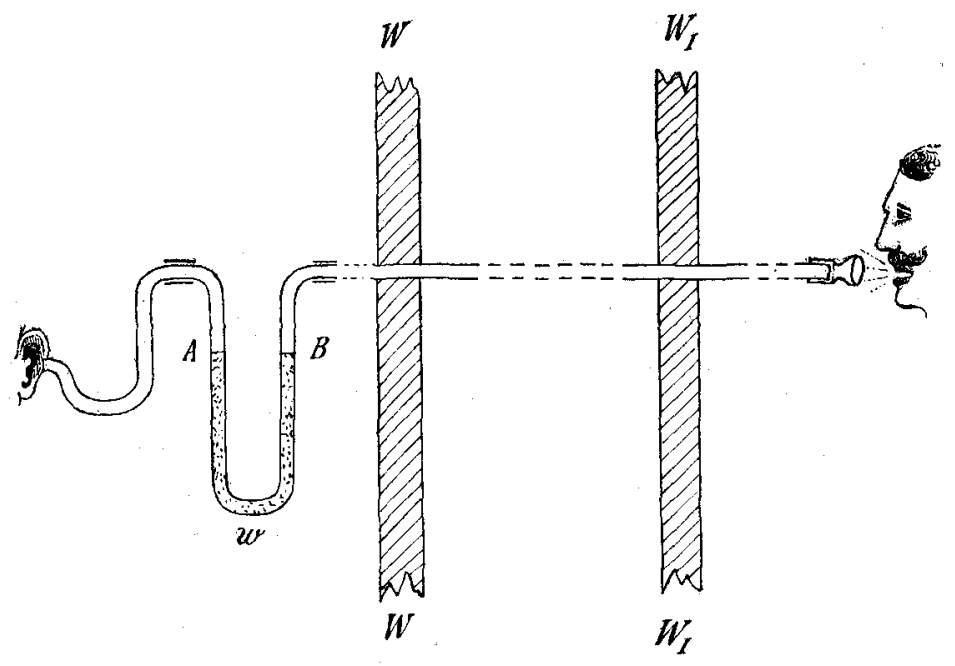

Fig. 2.

dessen horizontaler Schenkel $2 \mathrm{~cm}$ und dessen verticale Schenkel je $25 \mathrm{~cm}$ lang waren, während sein lichter Durchmesser $8 \mathrm{~mm}$ betrug. Eine Hauptbedingung für die Versuche war natürlich die, dass der 
Hörende von den zu untersuchenden Tönen und Vocalen nur das hören konnte, was den Widerstand passirt hatte, also eine directe Gehörswahrnehmung, etwa vermittelst des anderen Ohres durch die Luft und durch die Wände vollkommen ausgeschlossen war. Es musste daher die Tonquelle von dem Hörenden genügend weit entfernt und dieser von jener durch dichte Wände geschieden sein, welche einer directen Weiterleitung des Tones ausreichenden Widerstand entgegensetzten.

Die Versuchsanordnung gestaltete sich hiernach folgendermaassen:

In dem einen Zimmer (es ist das Zimmer $A$ des Versuches auf S. 309) wurde der U förmige Widerstandsapparat $A w B$ (s. Fig. 2) in passender Höhe auf einem mit nach der Höhe verstellbarer Platte versehenen Tisch aufgestellt. An seinem einen Ende $A$ ist ein dickwandiger Gummischlauch von $50 \mathrm{~cm}$ Länge angebracht, der wiederum in einen olivenförmigen, in den äusseren Gehörgang des Hörenden passenden Ansatz endigt. Das andere Ohr des Beobachters wurde mit dem Finger oder Wattepfropfen dicht versehlossen. An das andere Ende $B$ des Widerstandsapparates ist vermittelst eines kurzen, dicken Kautschukstückchens eine gegen $11 \mathrm{~m}$ lange Leitung von Messingröhren angeschlossen, welche, die Wände $W W$ und $W_{1} W_{1}$ eines zweiten Zimmers (Zimmer $B$ der Fig. 1) durchbrechend, in gerader Richtung in ein drittes Zimmer (Zimmer $C$ der Fig. 1) führt und dort etwa $3 \mathrm{~m}$ von der Wand entfernt in eine trichterförmige Erweiterung endigt, welche zur Aufnahme der Töne der in $20 \mathrm{~cm}$ Entfernung von ihr befindlichen Tonquellen dient. Die Röhrenleitung besteht aus drei innen glatten Messingröhren von durchschnittlich je $13 \mathrm{~m}$ Länge und $10 \mathrm{~mm}$ lichter Weite. Die Röhren sind unter sich durch kurze, dicke Kautschukstückchen so verbunden, dass Knickungen und Biegungen völlig vermieden werden. In dieser geraden, horizontalen Lage werden sie durch passende Stützen gehalten. Die für die Rohrleitung vorgesehenen Oeffnungen in den Wänden $W W$ und $W_{1} W_{1}$ sind um die Röhren herum dicht mit Watte verstopft, die Verbindungsthüren der betreffenden Zimmer geschlossen, sowie beiderseits mit dicken Teppichen behängt. So wird erreicht, dass der Hörende, dessen freies $\mathrm{Ohr}$ mit dem Finger oder passendem Wattepfropf verschlossen wird, selbst bei lautem Rufen nur das hören kann, was die Röhrenleitung passirt hat.

Zunächst wurden Versuche dahingehend angestellt, inwieweit Töne verschiedener Höhe, aber subjectiv gleich gross erscheinender 
Intensität durch die verschiedenen Widerstände beeinflusst werden. Als Tonquelle wurde eine Violine benutzt, und zwar wurde ein sehr gleichmässig ausgespieltes, altitalienisches Exemplar edler Bauart verwendet. Der Spieler stellte sich so vor dem Schallbecher der Leitung auf, dass die Oberdecke der Violine, die ja in erster Linie als resonirend in Betracht kommt, diesem zugewendet war, so zwar, dass die Längsachse der Violine horizontal und senkrecht zur Leitung stand, deren Verlängerung in etwa $20 \mathrm{~cm}$ Entfernung vom Schallbecher die Gegend des Stegs traf. Der Spieler war so geübt, dass er eine Tonleiter in subjectiv stets gleichbleibender Tonstärke zu spielen vermochte. Ein geübter Musiker war dem Spieler zur Controle beigegeben.

\section{Versuch 1.}

Das U-Rohr bleibt leer, so dass also die Fortleitung des Tones nur durch den Luftwiderstand und die verschiedenen Reflexionen an den Biegungsstellen und an den Verkuppelungen der Röhren beeinflusst warde.

Gespielt wird in mittleren $\mathrm{mf}$ eine Tonleiter von drei Octaven, von $g$ (mit 198 Schwingungen) bis $g^{\prime \prime \prime}$ (mit 1584 Schwingungen), und zwar so, dass jeder Ton auf einen langsam ausgehaltenen Bogenstrich kommt, und, wie oben erwähnt, in stets gleich erscheinender Tonintensität. Stellung des Spielers und Haltung der Violine ist oben geschildert.

Gehört wird die Tonleiter durch das leere Rohr in ganz regelmässigem Decrescendo, so dass jeder folgende Ton um etwas schwächer erscheint als der vorhergehende. Während die tieferen Töne sehr laut und voll geradezu an's Ohr schlagen, werden die höheren Töne, etwa von $d^{\prime \prime}$ ab (594 Schwingungen), nur wie aus der Ferne vernommen. Von $d^{\prime \prime \prime}(1188)$ ab wird fast nichts mehr gehört. Das regelmässige Decrescendo wird nur von einigen Tönen unterbrochen, nämlich denjenigen, bei denen leere Saiten der Violine mitschwingen können, also $g^{\prime} g^{\prime}$ und $g^{\prime \prime \prime}$, sowie $d^{\prime \prime} d^{\prime \prime \prime}, a a^{\prime \prime}, e^{\prime}$ und $\epsilon^{\prime \prime \prime}$. Diese klingen etwas lauter. Im speciellen Fall resp. bei der speciell verwendeten Violine waren es namentlich die verschiedenen $g\left(g^{\prime}, g^{\prime \prime}\right.$ und $\left.g^{\prime \prime \prime}\right)$, die etwas lauter klangen, so dass, während bei gespieltem $f^{\prime \prime \prime}$ kaum etwas gehört werden konnte, bei $g^{\prime \prime \prime}$ wieder eine geringe Tonwahrnehmung eintrat, die aber auch verschwand, wenn durch den aufgesetzten Finger die leere $g$-Seite am Mitschwingen verhindert wurde.

\section{Versuch 2.}

Die Versuchsanordnung bleibt dieselbe wie bei Versuch 1. Gespielt wird diesmal lauter, also in gleichmässigem Forte eine Tonleiter in 4 Octaven von $g$ bis $g^{\prime \prime \prime \prime}$ (198-3168 Schwingungen), sonst in gleicher Weise wie bei Versuch 1. Die leeren Saiten werden in den höheren Lagen durch die aufgesetzten Finger eines Assistenten am Mitschwingen verhindert.

Gehört wird die ganze Tonleiter und zwar wie beim 1. Versuch in ganz regelmässigem Decrescendo (ohne Störungen, da diesmal die leeren Saiten theils E. Pflüger, Archiv für Physiologie. Bd. 100. 
durch den Spieler selbst, theils durch den Assistenten am Mitschwingen verhindert wurden). Dieses regelmässige Decrescendo erstreckte sich von den tiefen Tönen, die diesmal sehr kräftig an's Ohr schlugen, bis etwa zum dreigestrichenen $d\left(d^{\prime \prime \prime}=1188\right.$ Schwingungen). Dieses wurde mit geringster Intensität und angespannter Aufmerksamkeit gerade noch wahrgenommen. Die folgenden Töne (bis $g^{\prime \prime \prime \prime}$ mit 3168 Schwingungen) zeigten keine wesentliche Intensitätsabnahme mehr, mit Ausnahme der beiden Töne $f^{\prime \prime \prime \prime}$ und $g^{\prime \prime \prime \prime}$, da diese beiden Töne vom Spieler meist nicht mehr mit derselben Intensität hervorgebracht werden konnten wie die vorhergehenden.

In einer Curve dargestellt, würden also die Empfindungen oder Lautheiten der verschieden hohen Töne eine etwa geradlinige absteigende Linie darstellen, die bei den tiefsten am böchsten und bei den höchsten am tiefsten liegt, wenn man (siehe Fig. 3) die Tonhöhen als Abscissen und die ihnen entsprechenden Empfindungen als Ordinaten verzeichnete. In den höheren Tönen von 1000-3000 Schwingungen verlief die Curve nahezu der Abscisse parallel.

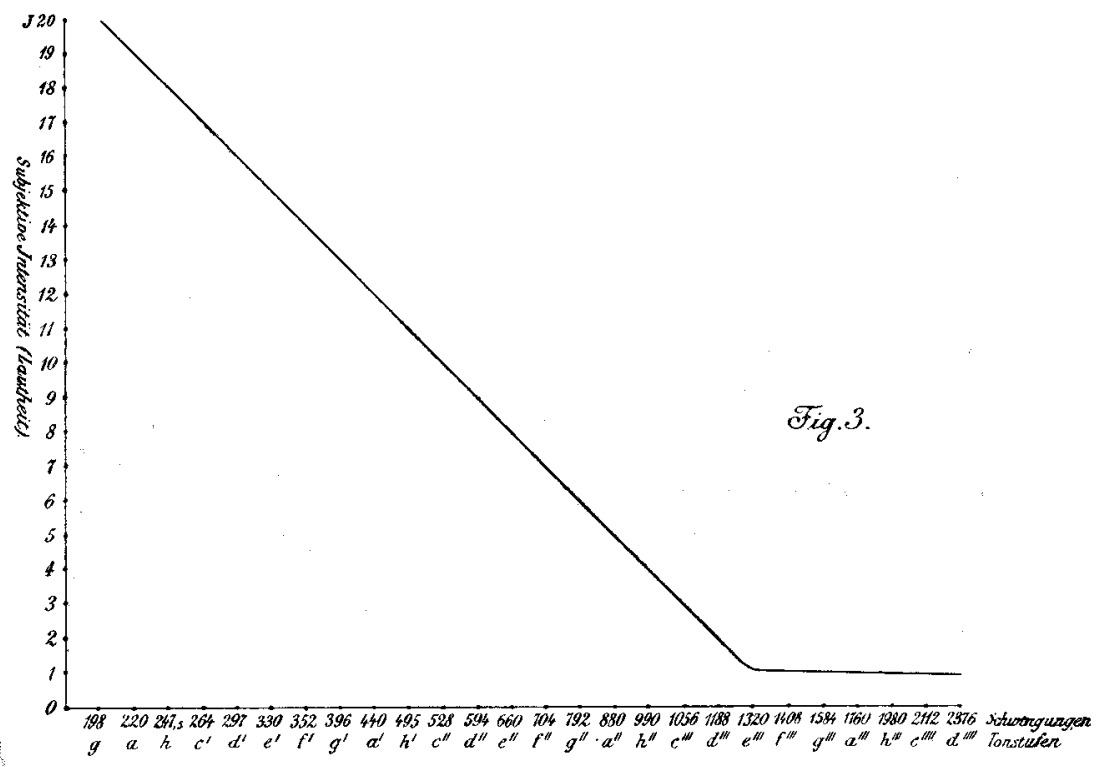

Bezüglich dieser Töne $d^{\prime \prime \prime}-g^{\prime \prime \prime \prime}$ von 1188-3168 Schwingungen, die, wie oben erwähnt, in annähernd gleicher Lautheit dureh die Rohrleitung gehört wurden, und zwar so, dass sie alle bei angespannter Aufmerksamkeit gerade noch wahrgenommen werden konnten, ist noch Folgendes zu bemerken. Bei ihnen allen wurde nämlich beobachtet, dass sie für das Ohr zeitweise verschwanden und 
dann wieder gehört wurden, sei es nun, dass diese Erscheinung durch schwankende Intensität von Seiten des Spielers veranlasst war, oder dass es sich hier um ein gleiches Phänomen handelte, wie es Urbantschitsch ${ }^{1}$ ) und später Hessler ${ }^{2}$ ) beim Abklingen von Stimmgabeln gefunden haben.

\section{Versuch 3.}

Gespielt wird in derselben Weise wie bei Versuch 2. Das U-förmige Rohr wird mit steigenden Mengen Schrots von $2 \mathrm{~mm}$ Durchmesser beschickt. Jedes Mal werden die in gleicher subjectiver Stärke gespielten Töne (von $g-g^{\prime \prime \prime \prime}$ ) in regelmässigem Decrescendo gehört.

Bei $5 \mathrm{ccm}$ Schrotfüllung wird von $e^{\prime \prime \prime}$ an nichts mehr gehört. Mit $10 \mathrm{ccm}$ Schrotfüllung ist der zuletzt gehörte Ton $h^{\prime \prime}$, mit $20 \mathrm{ccm}$ Füllung $e^{\prime \prime}$, mit $40 \mathrm{ccm}$ Füllung $h^{\prime}$. Bei den letztgehörten Tönen konnte wieder vielfach die oben erwähnte Erscheinung des Verschwindens und Wiederauftretens des Tons wahrgenommen werden, was natürlich andererseits die Feststellung eines Tones als des letzten gerade noch wahrnehmbaren vielfach erschwerte. Merkwürdiger Weise erhielten sich bei manchen Tönen, die den betreffenden Widerstand nicht mehr zu überwinden vermochten, vielfach die bei ihrer Erzeugung entstehenden Nebengeräusche, wie das rauschende Reiben des Bogens auf der Saite u. s. w., so dass letztere oft noch wahrgenommen werden konnten, während vom erzeugten Tone selbst nichts mehr gehört wurde.

\section{Versuch 4.}

Uebereinstimmende Resultate wurden mit hölzernen, offenen Labialpfeifen erzielt. Diese wurden mit dem Munde angeblasen und so gehalten, dass ihre Längsachse in der Verlängerung der Rohrleitung stand und ihr oberes Ende in $10 \mathrm{~cm}$ Abstand von der trichterförmigen Erweiterung der Leitung sich befand. Die von R. Kö nig in Paris stammenden Pfeifen standen uns in dem Tonumfang von $C-c^{\prime \prime \prime}$ zur Verfügung. Als Widerstand befand sich in dem U-Rohr $10 \mathrm{ccm}$ Schrot von $2 \mathrm{~mm}$ Durchmesser. Auch hier wurden durch den Widerstand hindurch die Töne in nach der Höhe zu stetig abnehmender Intensität gehört, wenngleich die Abnahme nicht so regelmässig erschien wie bei den Tönen der Violine (Versuch 2), da es bei den Pfeifen viel schwieriger war, erstens die einzelnen Töne in gleich erscheinender Intensität zu blasen, zweitens den angeblasenen Ton in gleicher Intensität auszuhalten. Beide Forderungen konnten von dem geübten Violinspieler auf der Violine viel präciser gelöst werden. Jedoch waren namentlich die Extreme ausserordentlich auffallend; denn während der Ton der angeblasenen $H$-Pfeife durch Leitung und Widerstand von $10 \mathrm{ccm}$ Schrot ganz laut gehört wurde, konnte von einer mit aller Kraft angeblassenen $h^{\prime \prime \prime}$-Pfeife,

1) Urbantschitsch, Ueber eine Eigenthümlichkeit der Schallempfindung geringster Intensität. Centralbl. f. med. Wissensch. 1875 Nr. 35.

2) V. Hessler, Archiv für Ohrenheilkunde Bd. 18 S. $234 \mathrm{ff}$. 
die für den Nahestehenden einen unerträglich lauten und durchdringenden Ton gab, selbst durch die leere Leitung nichts vernommen werden.

Ganz ähnlich dem Vorhergehenden waren die Resultate, wie sie bei Versuchen in freier Luft gewonnen wurden. Ort des Versuchs, sowie die äusseren Anordnungen waren dieselben, wie sie schon oben, bei den Versuchen über Abschwächung von Vocalklängen in freier Luft, beschrieben sind; nur wurde diesmal statt des Sängers ein Violinspieler aufgestellt, und zwar wiederum so, dass stets die Oberdecke der Violine dem Hörer zugewendet war. Im Uebrigen wurde in derselben Art gespielt wie in den eben geschilderten Versuchen. Die in dieser Weise vielfach wiederholten Versuche - es waren diese Wiederholungen wegen der im Freien stets auftretenden Momente, wie wechselnder Wind, Nebengeräusch u. s. w., nöthig liessen zwar eben dieser stets wechselnden Störungen wegen für die Hörbarkeit der einzelnen Töne keine unveränderlichen Entfernungen festlegen, aber jedes Mal dies mit Sicherheit erkennen, dass die tiefen Töne der Violine noch in grösserer Entfernung gehört werdeu konnten als die hohen, oder, was dasselbe ist, dass bei unveränderter Entfernung zwischen Hörer und Spieler die von Letzterem in gleicher subjectiver Stärke gespielte aufsteigende Tonleiter vom $g$ der $g$-Saite bis zum $g$ der $e$-Saite von Ersterem in gleichmässigem Decrescendo gehört wurde.

\section{Abschwächung von Vocalklängen durch poröse Medien und durch Luft.}

Dieselbe Versuchsanordnung wie in Versuch $1-4$ wurde benutzt, um den Einfluss der verschiedenen Widerstände auf die Gehörswahrnehmung der Vocale zu prüfen. Der Sänger steilte sich hierbei so auf, dass sein Mund von der in Mundhöhe befindlichen trichterförmigen Erweiterung der Rohrleitung etwa $20 \mathrm{~cm}$ in der Verlängerung der Leitung entfernt war. Gesungen wurden die einzelnen Vocale, wie bei den Versuchen in freier Luft, mit mittelstarker Stimme in verschiedenen, jeweils natürlich gleichen Tonhöhen, gleicher Tondaner und dem Sänger subjectiv gleich erscheinenden Tonstärken. Ein musikalisch geübter Assistent war dem Sänger wieder zur Controle beigegeben. Die einzelnen Vocale wurden, besonders wenn stärkere Widerstände angewandt wurden, dem Hörenden allemal vorher durch ein Klopfsignal angekündigt. 
Versuch 5.

Als Widerstand werden $30 \mathrm{ccm}$ Schrot von $2 \mathrm{~mm}$ Caliber verwendet. Die Vocale werden auf $g \frac{\sqrt{\ddagger}}{\square}$ gesungen. Sie werden alle durch den Widerstand deutlich verstanden, jedoch kommen sie mit sehr verschiedener Intensität an, so dass sie wieder in die schon mebrfach erwähnte Reihe $A, O, E, I, U$ geordnet werden können.

\section{Versuch 6.}

Als Widerstand werden $25 \mathrm{ccm}$ grobgepulverten Glases benutzt, dessen einzelne Körner ein durchschnittliches Caliber von $1 / 2 \mathrm{~mm}$ besitzen. Dieser Widerstand verhält sich nach $\mathrm{Tufts}^{1}$ ) zu dem beim vorigen Versuch benutzten etwa wie 10:8, muss also eine bedeutend stärkere Schallabschwächung verursachen. Die Vocale werden in mittelstarker Stimme auf $g \frac{\sqrt{\frac{1}{\ddagger}}}{4}$ gesungen.

\begin{tabular}{c|l}
\hline \hline $\begin{array}{c}\text { Gesungen } \\
\text { von R. L. }\end{array}$ & \multicolumn{1}{|c}{ Gehört von M. F. } \\
\hline$A$ & $A$ laut \\
$E$ & $E$ Schwaches $O$ \\
$E$ & U sehr schwach $\}$ mehr wie ein \\
$U$ & $I$ sehr schwach $\}$ dumpfer Ton \\
$U$ & $I$ \\
$I$ & $O$ ziemlich laut \\
$O$ & $U$ schwach und dumpf \\
$I$ & Ton \\
$U$ & $E$ ziemlich schwach \\
$E$ & Schwaches $O$ \\
$E$ & $E$ oder $I$ sehr schwach \\
$U$ & $A$ laut \\
$A$ & $U$ \\
$I$ & $U$ dumpfer Ton \\
$I$ & $?$ \\
$I$ & $A$ \\
$A$ & $O$ schwach \\
$E$ &
\end{tabular}

\begin{tabular}{l|l}
\hline Ges. v. Sch. \\
auf
\end{tabular}

1) F. L. Tufts, The transmission of Sound through porous materials. American Journal of science Voi. 11 Art. 29 S. 357. 1901. 


\begin{tabular}{|c|c|}
\hline Ges. von Sch. & Gehört von M. F. \\
\hline $\begin{array}{l}E \\
O \\
O \\
E \\
I \\
A \\
O \\
\\
E \\
A \\
U \\
I \\
A\end{array}$ & $\begin{array}{l}E \text { oder } O \text { schwach } \\
E \text { ziemlich stark } \\
O \text { ziemlich stark } \\
E \text { schwach } \\
I \text { und } U \text { schwach und unbestimmt } \\
A \text { stark } \\
O \text { ? ziemlich stark, aber ohne dent- } \\
\text { lichen Charakter } \\
E \text { ? schwach u. ohne dentl. Charakter } \\
A \text { stark } \\
U \text { dumpf } \\
U \text { dumpf } \\
A \text { stark }\end{array}$ \\
\hline
\end{tabular}

\section{Versuch 7.}

Der Widerstand wird noch um $5 \mathrm{ccm}$ erhöht, so dass sich nunmehr $30 \mathrm{ccm}$ von dem grobgepulverten Glas in dem U-Rohr befinden.

Tonhöhe $g$ 年

\begin{tabular}{|c|c|}
\hline $\begin{array}{l}\text { Vocale ges. } \\
\text { von R. L. }\end{array}$ & Gehört von M. F. \\
\hline $\begin{array}{l}A \\
E \\
A \\
O \\
U \\
I \\
A \\
E \\
O \\
A\end{array}$ & $\begin{array}{l}\text { A laut und deutlich } \\
\text { Unbestimmt, schwacher, hohler Ton } \\
A \text { deutlich } \\
Z \text { iemlich lauter Ton, } E \text { oder } O ? \\
U \text { sehr dumpf } \\
I \text { oder } U \text { ? } \\
A \\
\text { Unbestimmter Ton, schwach } \\
\text { Ebenso, aber stärker } \\
A \text {. }\end{array}$ \\
\hline
\end{tabular}

\section{Versuch 8.}

Die Versuchsanordnung bleibt die gleiche. Gesungen wird in

\begin{tabular}{|c|c|}
\hline $\begin{array}{c}\text { Vocale ges. } \\
\text { von R. L. }\end{array}$ & Gehört von M. F. \\
\hline $\begin{array}{l}A \\
U \\
I \\
A \\
O\end{array}$ & $\begin{array}{l}A \\
\text { Keine Wahrnehmung } \\
\text { Dumpfer Ton, I? } \\
A \text { deutlich und laut } \\
\text { Lauter, hohler Ton ohne deutlichen } \\
\quad \text { Vocalcharakter }\end{array}$ \\
\hline
\end{tabular}




\begin{tabular}{c|l}
\hline $\begin{array}{c}\text { Vokale ges. } \\
\text { von R. L. }\end{array}$ & Gehört von M. F. \\
\hline$E$ & Schwach, $O$ oder $E$ ? \\
$I$ & Dumpf und schwach, $U$ oder $I ?$ \\
$A$ & $A$ \\
$E$ & $O$ \\
$U$ & $U$ \\
$U$ & $I$ \\
$A$ & $A$
\end{tabular}

\section{Versuch 9.}

Bei gleichbleibender Versuchsanordnung wird wiederum in $e^{\prime}$ sungen. Dazwischen wird von Zeit zu Zeit eine offene hölzerne Labialpfeife, die ebenfalls den Ton $e^{\prime}$ gibt, mit einer den gesungenen Vocalen möglichst gleichen Lautheit angeblasen.

Während, wie auch in den vorhergehenden Versuchen, $A$ stets deutlich erkannt wird, auch $O$ und $E$ gegenüber dem Pfeifenton sich noch als gesungene Vocale charakterisiren, (wenn auch beide einander sehr ähnlich werden, und zwar so, dass für das beobachtende $\mathrm{Ohr} O$ dasselbe Klangbild in grösserer Lautheit darstellt, welches $E$ in schwächerer gibt), so erscheint bei $U$ und $I$ der specifische Vocalcharakter derart zerstört, dass der Hörer eine sichere Unterscheidung zwischen $U$ und $I$ und dem Pfeifenton nicht mehr zu treffen vermag. (Vgl. folgende Tabelle.)

\begin{tabular}{|c|c|}
\hline $\begin{array}{l}\text { Vocale gesungen } \\
\text { und Töne geblasen } \\
\text { in } e^{\prime} \text { von R. L. }\end{array}$ & Gehört von M. F. \\
\hline $\begin{array}{c}A \\
A \\
I \\
\\
\text { Pfeifenton } \\
A \\
O \\
E \\
\text { Pfeifenton } \\
I \\
A \\
E^{\top} \\
O \\
A \\
U \\
\text { Pfeifenton } \\
A \\
E \\
O \\
A\end{array}$ & $\begin{array}{l}A \text { laut und deutlich } \\
A \text { laut und deutlich } \\
U \text { ? } \\
\text { Ton } \\
U \text { oder } I ? \\
A \\
O \\
\text { Schwaches } O \\
\text { Dumpfer Ton, vielleicht } U \\
\text { Ebenso } \\
A \\
E \text { oder } O \\
O \\
A \\
\text { Dumpfer Ton, vielleicht } U \\
U ? \\
A \\
I ? \\
E \text { oder } O \\
A\end{array}$ \\
\hline
\end{tabular}




\section{Versuch 10.}

Der Widerstand wird noch weiter verstärkt, indem statt der verwendeten groben Massen $6 \mathrm{ccm}$ feingesiebten Flusssandes in das U-Rohr eingefüllt werden. Im Uebrigen bleiben die Verhältnisse wie beim vorhergehenden Versuch; also zwischen die gesungenen Vocale werden wieder Pfeifentöne von gleicher Tonhöhe und Lautheit eingeschaltet.

Die benutzte Sandmenge setzt der Schallleitung einen sehr starken Widerstand entgegen, so dass vom gesungenen Vocal $U$ manchmal gar nichts mehr gehört werden kann. Der Vocalcharakter der übrigen Vocale mit Ausnahme des $A$, welches nicht beeinflusst und stets richtig und deutlich erkannt wird, und in vereinzelten Fällen auch des $O$, das dann und wann noch etwas von seinem Charakter bewahrt, wird dagegen so völlig zerstört, dass diese sich unter einander so wenig wie von dem angeblasenen Pfeifenton unterscheiden lassen. Sie klingen alle wie ein ferner, hohler Ton und unterscheiden sich unter einander nur durch ihre verschiedene Stärke, indem der dem gesungenen $U$ entsprechende gehörte Ton (soweit er nicht ganz ausfällt) schwächer erscheint, als der dem $I$ entsprechende und dieser wieder hinter den $E$ und $O$ entsprechenden bezüglich der Lautheit zurücksteht. Letzterer Vocal wird übrigens, wie oben erwähnt, manchmal noch richtig erkannt. (Vgl. Tabellen.)

\begin{tabular}{|c|c|}
\hline $\begin{array}{l}\text { Vocale gesungen } \\
\text { und Töne geblasen } \\
\text { von } \mathrm{R} \text {. L. } \\
\text { in } e^{\prime}\end{array}$ & Gehört von M. F. \\
\hline $\begin{array}{c}A \\
O \\
\text { Pfeifenton } \\
U \\
I \\
E \\
O \\
A \\
I \\
U \\
O \\
\text { Pfeifenton } \\
A \\
E \\
I \\
A \\
O \\
U \\
I \\
\text { Pfeifenton } \\
E\end{array}$ & $\begin{array}{l}A \text { deutlich } \\
O \text { ? } \\
\text { Ton, vielleicht } O \\
\text { Keine Wahrnehmung } \\
\text { Schwacher Ton } \\
\text { Derselbe Ton, etwas stärker } \\
\text { Ebenso } \\
A \\
\text { Schwacher Ton, vielleicht } U \\
\text { Schwacher Ton } \\
\text { Ton, etwas stärker } \\
\text { Ebenso } \\
A \\
\text { Ton } \\
\text { Ton } \\
\text { A } \\
O \text { ? } \\
\text { Keine Wahrnehmung } \\
\text { Ton schwach } \\
\text { Ton etwas stärker } \\
\text { Ton wie der vorhergehende }\end{array}$ \\
\hline
\end{tabular}

Zusammengefasst sind die Resultate der Versuche mit gesungenen Vocalen folgende: 
Ganz entsprechend den bei den Versuchen in freier Luft erhaltenen Resultaten wurden durch die hier verwendeten Widerstände (sei es, dass es sich nur um den Widerstand der in der Rohrleitung eingeschlossenen Luftsäule, verbunden mit den durch Reflexionen exzeugten geringfügigen Störungen, handelte, sei es, dass körnige Materialien, wie Schrot, Glaspulver oder Sand verwendet wurden) die in subjectiv gleich gross erscheinender Tonintensität gesungenen Vocale derart beeinflusst, dass sie nach Passirung kleinerer Widerstände für den Beobachter in deutlich zu unterscheidenden Lautheiten ankamen, welche wiederum, nach zunehmender Intensität geordnet, die Reihe $U, I, E, O, A$ ergaben.

Zugleich wurden die Vocalcharaktere derart angegriffen und durch grössere Widerstände zerstört, dass $U$ und $I$, bei höheren Widerständen auch $O$ und $E$ je einander immer ähnlicher im Klang erschienen und daher häufig mit einander verwechselt wurden, bis schliesslich die ersten beiden nur noch als dumpfer, die letzten nur als hohler Klang wahrgenommen wurden und von einem Pfeifenton gleicher Tonböhe nicht mehr unterschieden werden konnten (siehe Versuche 9 u. 10). Niemals dagegen angegriffen, sondern in allen Fällen laut und deutlich erkannt wurde das $A$.

Alle oben mitgetheilten Versuche wurden in den verschiedensten Tonhöhen nachgeprüft. Die Resultate waren stets übereinstimmend, nur mit dem Untersehied, dass, in je höheren Tonlagen die Vocale gesungen wurden, desto kleinere Widerstände schon genügten, um die beschriebenen Zerstörungen der Vocalcharaktere herbeizuführen, worin eine deutlich ausgesprochene Uebereinstimmung mit dem Verhalten verschieden hoher Töne gegenüber den Widerständen liegt (vgl. Versuch 1 bis 4).

Wie sich Geräusche und die verschiedenen in unserer Sprache angewendeten Consonanten gegenüber Widerständen verhalten, darüber habe ich leider wegen Zeitmangels keine Versuche anstellen können.

E. Versuche über die Beziehungen der Stärke (objectiven Energie) und der Lautheit (subjectiven Empfindung) von Klängen.

Die erste Frage, die sich uns bei unseren Versuchen aufwarf, war die: Wie kommt es, dass von zwei gleich lauten, aber verschieden hohen Klängen oder von zwei gleich lauten und gleich hohen Vocalen, 
z. B. von $A$ und von $U$, der eine Klang durch einen Widerstand stark geschwächt, ja, mitunter ganz vernichtet und unhörbar wurde, während der andere, ebenso laute nur wenig an seiner Lautheit und Art eingebüsst hatte? Wie verhielt es sich da mit der objectiven Stärke dieser Klänge? Wie gross war die Energie des einen oder anderen? Steckte in den höheren Klängen, weil sie durch die Widerstände so ausserordentlich stark geschwächt wurden, viel weniger Energie als in den tiefen? Oder war in einem $A$ viel mehr Energie enthalten als in einem gleich lauten $U$ ?

Diese Fragen sind sehr schwer, ja, vielfach kaum zu beantworten. Beginnen wir mit einigen einfachen Thatsachen. Nehmen wir zunächst einmal an, ein Klang von $n$ Schwingungen werde dadurch erzeugt, dass man mit einem Hämmerchen auf eine Unterlage $n$ unter einander gleich starke und selbstverständlich zeitlich gleich abständige Schläge führe. Verdoppelt man jetzt die Zahl dieser Schläge, und nimmt man ferner der Einfachheit halber an, die gesammte Energie der Schläge setze sich in Klang um, so ist es ohne Weiteres klar, dass in dem Klang von $2 n$ Schwingungen für gleiche Zeiten die doppelte Energie enthalten ist als in dem Klang von $n$ Sehwingungen. Es wird desshalb auch Jedermann ziemlich natürlich finden, dass der zweite, höhere Ton viel stärker - ungefähr zwei Mal so stark - auf unser Ohr wirkt als der erste, gerade so, wie sich Niemand wundern wird, dass drei Schläge mit einer Gerte innerbalb einer bestimmten kurzen Zeit etwa drei Mal so viel Schmerzen bereiten als ein einziger Schlag. Ja, man wird sogar im Allgemeinen geneigt sein, anzunehmen, dass die Grösse der Schmerzen im zweiten Fall mehr als drei Mal so stark sein dürfte.

Derartige, oben erwähnte Klänge erhält $\operatorname{man}$ z. B., wenn man eine einfache, durch ein Uhrwerk gleichmässig angetriebene Sirenenscheibe vermittelst eines zweckmässig gestalteten Rohres in stets gleicher Art und Kraft anbläst. Jeder einzelne Luftstoss der verschiedenen, auf diese Weise erzeugten Töne dürfte dem anderen an Energie nahezu gleich sein. In dem einen Fall wiederholen sich die Stösse nur viel schneller als in dem anderen. Auch ein gegen gezahnte, sich drehende Räder gehaltenes Kartenblatt gibt wohl derartige Klänge, und aller Wahrscheinlichkeit nach erzeugen sie auch gewisse Blasinstrumente (z. B. Fagott, Clarinette), deren Töne nach den Untersuchungen von 
Grützner ${ }^{1}$ ) sich aus nahezu gleich starken, aber verschieden häufigen Stössen zusammensetzen.

Dass unter solchen Umständen die höheren Klänge sehr viel lauter ${ }^{2}$ ) klingen als die tiefen, wird Niemanden verwundern; denn sie enthalten ja um so mehr Energie in der Zeiteinbeit, je höher sie $\sin$.

Aber auch wenn man verschieden hohe Töne in gleicher Zeitdauer derart erkikingen lässt, dass in einen hohen Ton in der Zeiteinheit dieselbe Gesammtenergiemenge hineingelegt wird wie in einen tiefen, so scheint uns doch der hohe Ton viel lauter zu sein. Einen derartigen lehrreichen Versuch hat $\mathrm{Helmholtz}{ }^{3}$ ) mit seiner Doppelsirene angestellt. Er sagt hierüber Folgendes: „Es ist von mebreren Akustikern zuweilen die Voraussetzung ausgesprochen worden, dass unter übrigens gleichen Umständen die Stärke der Töne verschiedener Höhe der lebendigen Kraft der Luftbewegung direct proportional sei oder, was auf dasselbe herauskommt, der Grösse der zu ihrer Hervorbringung aufgewandten mechanischen Arbeit; aber ein einfacher Versuch mit der Sirene zeigt, dass, wenn die gleiche mechanische Arbeit aufgewendet wird, um tiefe oder hohe Töne unter übrigens gleichen Verhältnissen zu erzeugen, die hohen Töne eine ausserordentlich viel stärkere Empfindung hervorrufen als die tiefen. Wenn man nämlich die Sirene durch einen Blasebalg anbläst, so dass ihre Scheibe immer schneller und schneller umläuft, und wenn man dabei darauf achtet, die Bewegung des Blasebalgs ganz gleichmässig zu unterhalten, so dass sein Hebel gleich oft in der Minute und immer um dieselbe Grösse gehoben wird, wobei denn auch der Balg gleichmässig gefüllt bleibt und immer dieselbe Menge Luft unter gleichem Druck in die Sirene getrieben wird: so hat man anfangs, solange die Sirene langsam läuft, einen schwachen, tiefen Ton, der immer höher und höher wird, dabei aber gleichzeitig an Stärke ausserordentlich zunimmt, so dass die höchsten Töne von etwa 880 Schwingungen, die ich auf meiner Doppelsirene hervorbringe, eine kaum ertragbare

1) P. Grützner, Stimme und Sprache, in Hermann's Handbuch der Physiologie Bd. 1 H. 2 S. 28.

2) Ich gebrauche das Wort "laut" und "Lautheit" für die subjectiven und, wenn nichts Besonderes hinzugefügt, "stark" und "Stärke" für die objectiven Eigenschaften eines Klangs.

3) H. Helmholtz, Die Lehre von den Tonempfindungen, 5. Aufl., S. 290. Braunschweig 1896. 
Stärke haben. Hierbei wird fortdauernd bei Weitem der grösste Theil der sich gleichbleibenden mechanischen Arbeit auf die Erzeugung der Schallbewegung verwendet, und nur ein kleiner Theil kann durch die Reibung der umlaufenden Scheibe in ihren Acbsenlagern und durch die mit ihr in Wirbelbewegung gesetzte Luft verloren gehen. Diese Verluste müssen bei schneller Rotation grösser werden als bei langsamer, so dass für die Hervorbringung der hohen Töne sogar weniger Arbeitskraft übrig bleibt als für die tiefen; und doch erscheinen in der Empfindung die hohen Töne so ausserordentlich viel stärker als die tiefen Töne."

Dieser Helmholtz'sche Sirenenversuch ist also anders als die oben erwähnten, nämlich als der imaginäre Klopfversuch mit dem Hämmerchen und die Versuche mit den ganz einfachen Sirenen. In der Helmholtz'schen Sirene werden ja die einzelnen Stösse um so schwächer, je häufiger sie auf einander folgen; sie sind also der Schwingungszahl der erzeugten Klänge umgekehrt proportional. In dem einen Fall, beim tiefen Ton, treffen unser Ohr wenige starke, in dem anderen, beim hohen Ton, viele schwache Schläge; ihre Gesammtenergiemengen in gleichen Zeiten aber sind einander gleich.

Nun sind in neuerer Zeit (nach Abschluss meiner Arbeit) über die Empfindlichkeit des menschlichen Ohres für Töne verschiedener Höhe interessante und wichtige Untersuchungen veröffentlicht worden; insonderheit hat man sich die von $\mathrm{Helmholtz}$ angeregte, oben erwähnte Frage zu lösen bemüht, ob dieselbe Energiemenge, zur Erzeugung von hohen oder von tiefen Tönen verwendet, auch subjectiv gleich laute Töne erzeugt. Wenn nun auch im Einzelnen die Angaben der verschiedenen Forscher $\left.\left.{ }^{1}\right)^{2}\right)^{3}$ ) weit auseinandergehen, darin stimmen sie alle mit Helmholtz überein, dass, kurz gesagt, das menschliche Ohr für tiefe Töne ausserordentlich viel weniger

1) H. Zwaardemaker u. F. H. Quix, Schwellenwerth u. Tonhöhe. Engelmann's Arch. f. Physiol. 1902 Supplement S. 367.

2) P. Ostmann, Schwingungszahlen und Schwellenwerthe. Ebenda 1908 S. 321 .

3) M. Wi n, Ueber die Empfindlichkeit des menschlichen Ohres für Töne verschiedener Höhe. Pflüger's Archiv Bd. 97 S. 1. 1908. - Vgl. ausserdem: P. Ostmann, Ein objectives Hörmaass und seine Anwendung. Wiesbaden 1903, und L. Jacobson u. W. Cowl, Ueber die Darstellung und Messung der Schwingungsamplituden ausklingender Stimmgabeln mit Hülfe der "Linearkinematographie". Engelmann's Arch. f. Physiol. 1903 S. 1. 
empfindlich ist als für hohe Töne von gleicher objectiver Stärke. Ja, Wien behauptet auf Grund seiner sorgfältigen Versuche, dass ein (reiner) Ton von 50 Schwingungen, um eben wahrnehmbar zu sein, etwa 100 Millionen Mal mehr Energie besitzen müsse als ein Ton von 2000 Schwingungen. Für Töne von 1000 bis 5000 Schwingungen, das sind etwa die charakteristischen Töne der menschlichen Sprache, besteht eine nahezu gleiche, ausserordentlich grosse Empfindlichkeit. Darauf beginnt die Empfindlichkeit wieder langsam zu fallen.

Indem ich nun zu meinen Versuchen wieder zurückkehre, welche ich - wie gesagt - vor, beziehungsweise ohne Kenntniss der oben erwähnten Arbeiten angestellt habe, so suchte ich mir auf Anrathen von Herrn Prof. v. Grützner über die objective Stärke der von mir untersuchten Klänge wenigstens dadurch ein ungefähres Bild zu machen, dass ich ihre Amplitude, genauer gesagt die Amplitude einer empfindlichen, von ihnen angesprochenen Membran feststellte.

Hierzu diente mir die Membran eines König'schen Spiegels. Dieselbe war schon seit mehreren Jahren im Institut im Gebrauch und hielt sich in gleich vollkommener Weise auch jetzt noch. Sie bestand aus gutem, dünnem Briefpapier, welches auf den Rahmen der manometrischen Kapsel aufgeklebt und dann mit alkoholiseher Schellacklösung getränkt war.

Die Untersuchung erstreckte sich nur auf gesungene Vocale und nicht auch auf die gespielten Violintöne, da letztere sich viel zu schwach erwiesen, um im Spiegel ein deutliches Flammenbild zu erzeugen.

Die Versuchsanordnung gestaltet sich hiernach folgendermaassen: Auf einem Tisch wird in Mundhöhe des vor dem Tische sitzenden Sängers vermittelst einer passenden Stütze ein kleiner Schalltrichter aufgestellt, dessen eines Ende durch ein kurzes, dickwandiges Kautschukstück fest mit einem aus Messing gearbeiteten, innen durchaus glatten, Y-förmigen Röhrenstück verbunden ist, und zwar so, dass der eine Schenkel die Fortsetzung des Schalltrichters bildet, während die beiden anderen auseinandergehen und sich in ein U-förmiges Glasrohr fortsetzen, mit dem sie wiederum durch kleine Stückchen Kautschukschlauch fest verbunden sind. In einem der beiden U-Rohre befinden sich $5 \mathrm{ccm}$ Schrot, dessen einzelne Körner einen Durchmesser von $2 \mathrm{~mm}$ besitzen; das andere, durchaus gleiche U-Rohr ist leer. Die beiden anderen Enden der U-förmigen 
Glasröhren sind wiederum in derselben Weise mit den beiden winklig zusammenlaufenden Schenkeln eines Y-förmigen Messingrohres verbunden, dessen dritter Schenkel vermittelst eines längeren, festwandigen Kautschuksehlauchs mit der manometrischen Kapsel eines König'schen Apparates verbunden ist. Sein rotirender Spiegel ist so aufgestellt, dass er vom Sänger selbst gut beobachtet werden kann.

Es entstehen also für die Fortleitung des gesungenen Vocals zwei Wege von nebengezeichneter Form $-<{ }_{b}^{a}>-$. Der eine Weg führt durch das leere (nicht gezeichnete) U-Rohr $a$, der andere durch das mit Schrot angefüllte $b$, je nachdem man den nach $b$ oder $a$ führenden Schlauch mit einer Klemme oder dem Finger zudrückt. So ist es möglich, dass, während der Sänger einen Vocal aushält, dieser Vocal rasch nach einander ein Mal durch das leere Rohr und dann wieder durch den Schrotwiderstand mittelst eines Fingerdrucks eines Assistenten oder des Sängers selbst geleitet werden kann und so die jeweiligen, schnell eintretenden Veränderungen des Flammenbildes vom Sänger beobachtet und verglichen werden können.

Vorbedingung ist natürlich, dass der Sänger alie Vocale in möglichst gleicher Höhe und Lautheit singt und aushält, sowie dass der Schallbecher, der so ausgeschnitten war, dass er sich der Mundumgebung des Sängers anzupassen vermochte, immer in gleicher Weise an den Mund gesetzt und an diesen mit immer gleichem, leichtem Druck angelegt wird, was sich nach einiger Uebung leicht erreichen lässt; denn schon ein um Weniges stärkeres Andrücken, durch welches die Druckschwankungen im ganzen Röhrensystem gesteigert und damit natürlich auch die Flammenhöhen beeinflusst werden, fühlt man deutlich in den die Mundöffnung umgebenden Hautpartien. Der Schallbecher selbst ist so ausgeschnitten, dass er sich der Mundumgebung des Sängers überall gut anlegt. Der Spiegel wird von einem Assistenten in stets gleicher Geschwindigkeit gedreht.

Das ganze, innen durchweg glatte Röhrensystem hat überall eine lichte Weite von $8 \mathrm{~mm}$. Jeder der drei Schenkel der beiden Y-förmigen Röhren ist $10 \mathrm{~cm}$ lang; die Länge des auf- und absteigenden Schenkels beider U-Rohre beträgt je $20 \mathrm{~cm}$, die des Verbindungsstückes je $2 \mathrm{~cm}$. Es sind also sämmtliche Maasse bej den beiden oben beschriebenen, für die Fortleitung des gesungenen Vocals benutzten Wegen gleich gemacht; demnach dürften auch die 
Störungen und Fehlerquellen, wie sie durch die verschiedenen Reflexionen und Interferenzen sich ergeben, bei beiden Wegen so ziemlich dieselben, also zu vernachlässigen sein.

Nachdem der Experimentirende und der Assistirende durch oftmals wiederholte Versuche sich mit dem beschriebenen Apparat eingeübt hatten, so dass es sicher gelang, in möglichst gleicher Höhe und Lautheit zu singen, den betreffenden Vocal einige Zeit lang in ganz gleicher Art auszuhalten, den Schallbecher in stets gleicher Weise an den Mund anzulegen, sowie endlich den Spiegel stets in gleicher Weise rotiren zu lassen, wurden auch jedes Mal übereinstimmende Resultate erzielt, die ich im Folgenden wiedergebe und die ich und Andere sogar abzuzeichnen in der Lage waren.

Das Flammenbild des auf $c$ gesungenen Vocals $A$ wurde durch den eingeschalteten Widerstand nur sehr wenig verändert. Die gesammte Curve wurde etwas niedriger, dann und wann konnte auch eine oder die andere feinere Zacke der Curve nach eingeschaltetem Widerstand nicht mehr wahrgenommen werden, im Grossen und Ganzen blieb aber das charakteristische Bild der Curve so ziemlich unverändert.

Etwas mehr beeinflusst zeigen sich die Curven des Vocals $O$. Die hohe, fast senkrechte Zacke meiner Vocalbilder erscheint bedeutend niedriger und lange nicht so steil. Die die hohe Zacke in charakteristischer Weise nochmals einschneidende kleinere Zacke ist verschwunden; das ganze Bild nähert sich demjenigen, welcher der Vocal $E$ nach Passirung des Widerstands zeigt.

Beim Vocal $\boldsymbol{E}$ erscheint die ganze Curve nach Einschaltung des Widerstands bedeutend niedriger und mehr liegend. Fast alle kleineren Zacken sind verschwunden.

Ganz bedeutend beeinflusst, ja, beinahe zerstört erscheint das Flammenbild der Vocale $I$ und $U$.

Die regelmässigen Zacken des Vocals $I$ erseheinen nach Einschaltung des Widerstands fast ganz abgeflacht, ebenso werden die ohne Widerstand ganz besonders hoch und deutlich erscheinenden Zacken des Vocals $U$ ganz niedrig (was im höchsten Maasse überraschend und schwer verständlich ist), so dass bei beiden Vocalen nur noch kleine, ganz flache Erhebungen übrig bleiben und die beiden modificirten Curven sich von einander kaum unterscheiden.

Ganz entsprechend den auf $c^{\prime}$ gesungenen Vocalcurven waren die resultirenden Bilder bei anderen Tonhöhen ${ }^{1}$ ); nur genügten bei

1) Es wurde noch in $q, c i s^{\prime}, e^{\prime}, f^{\prime}$ u. $g^{\prime}$ gesungen. 
höheren Tonlagen schon kleinere Widerstände, um die entsprechenden Resultate zu erbalten.

Die geschilderten Veränderungen, welche die Flammenbilder namentlich der auf $c^{\prime}$ gesungenen Vocale durch den zu passirenden Widerstand erfuhren, möchte ich in Uebereinstimmung mit den bisherigen Versuchen so deuten, dass das $A$ durch den Widerstand in seiner Intensität zwar eine geringe Abschwächung erfährt (die Amplitude der Curve wird nur wenig vermindert), in seinem Vocalcharakter aber beinahe unbeeinflusst bleibt (die charakteristischen kleinen Zacken bleiben gut erhalten). Beim $O$ ist schon eine weitergehende $\Lambda$ mplitudenabschwächung zu constatiren (die steilen grossen Zacken werden niedriger), wie auch noch in etwas grösserem Maasse beim $E$. Beide Flammenbilder werden einander ähnlicher, wie ja auch die Vocale selbst.

Die Amplituden der Flammenbilder von $I$ und $U$ endlich werden durch den Widerstand ganz bedeutend vermindert, so dass nur noch ganz kleine, niedrige und rundliche Zacken zu sehen sind. In ihrem Charakter unterscheiden sie sich kaum mehr von einander, ja, gleichen einander oft ganz und gar.

Es würden demnach die hier auf mehr objectivem Wege gewonnenen Resultate den in den früheren Versuchen subjectiv erhaltenen entsprechen, wobei ich mir allerdings keineswegs verhehle, dass die Verhältnisse verwickelt und schwer zu übersehen sind. Indessen habe ich die Versuche so oft wiederholt, geprüft und von Anderen prüfen lassen, dass ich die oben beschriebenen Ergebnisse in den von mir untersuchten Fällen für gesichert halte.

Wenn ich mich nun schliesslich bemühe, die Ergebnisse meiner Versuche zu deuten, namentlich also zunächst zu erklären, warum tiefe Klänge durch Widerstände viel weniger geschwächt werden als gleich laute hohe, so ist zunächst die Frage zu beantworten: Wie steht es mit der Stärke, mit den in den verschiedenen Klängen enthaltenen objectiven Energiemengen einerseits, und wie verhält sich hierza die Grösse der Empfindung, welche durch die verschiedenen Töne in unserem Gehörorgan ausgelöst wird, andererseits? Die einfachste Klärung dieser Frage und der durch meine Versuche erhaltenen Resultate scheint mir, in Anlehnung an die oben (S. 324) erwäbnten Arbeiten, die Annahme zu bringen, dass in der That unser Gehörorgan durch die hohen von mir verwendeten Töne ausserordentlich viel stärker 
gereizt wird als durch die tiefen, dass eben das Organ für jene ausserordentlich viel mehr empfindlich ist als für diese. Diese Annahme ergibt für meine Versuche folgende Erklärung: Stelle ich einem geübten Violinspieler die Aufgabe, eine aufsteigende Tonleiter in drei Octaven von $g$ bis $g^{\prime \prime}$ in stets gleichbleibender (subjectiver) Stärke zu spielen, so wird er, da ja sein für die Erkennung kleiner Tonstärkeuntersehiede geübtes und empfindliches Ohr die richtige Ansführung dieser Aufgabe überwacht, nach der Höhe aufsteigend in demselben Maasse obj ecti v immer leiser spielen, $d . h$. um so geringere Tonenergiemengen liefern, als mit zunehmender Tonhöhe die Empfindlichkeit seines Gehörorgans für diese Töne wächst. Ein daneben stehender Beobachter wird natürlich von dieser aufsteigenden Tonleiter, deren Töne in ihrer objectiven Intensität fortwährend abnehmen, wieder den Eindruck gleichbleibender Tonstärke bekommen. Befindet sich nun aber zwischen Spieler und Hörer ein Widerstand, sei es freie, sei es in Rohrleitungen eingeschlossene Luft, sei es endlich Schrot u. s. w., so wird, wie ich zunächst der Einfachheit halber annehmen möehte, durch die betreffenden Widerstände immer gleich viel Schallenergie vernichtet, d. h. in Wärme oder anderweitige Bewegung umgesetz. Es wird also hierdurch das Verhältniss der objectiven Schallenergie der tiefen zu derjenigen der hohen Töne sehr zu Ungunsten der letzteren beeinflusst. Denn betrage z. B. die Energiemenge eines tiefen Tones 1000 , die eines ebenso lauten bohen 100 , und vernichte der Widerstand in beiden Fällen eine Energiemenge von 10, so wird jetzt aus dem Verhältniss 1000:100 das Verhältniss 990:90, mit anderen Worten: Der hohe Ton war obne Widerstand objectiv nur 10 Mal so schwach, als der gleichlaute tiefe. Jetzt nach Einschaltung des Widerstandes ist er 11 Mal so schwach, kann also nicht mehr gleichlaut, sondern muss leiser erklingen als der tiefe.

Hierzu kommt aber noch etwas Anderes, was die hohen Töne gegenüber den tiefen benachtheiligt, selbst wenn sie vor der Passirung der Widerstände gleich grosse Energiemengen besässen wie die tiefen. Gleichwie nämlich die kurzwelligen violetten Strahlen schwerer trübe Medien durchsetzen als die langwelligen rothen, wie ferner auch die

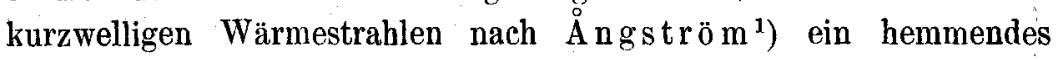
Medium schwerer durchsetzen als die langwelligen Strahlen („Das

1) K. Ångström, Beobacht. über die Durchstrahlung von Wärme versch. Wellenlänge u. s. w. Annalen der Physik und Chemie Bd. 36 S. 715. 1889. 
Medium ist mehr durchsichtig, je grösser die Wellenlänge ist", sagt Ångström), so ähnlich verbält es sich nach $\mathrm{Helmholtz}{ }^{1}$ ) auch mit sehr hohen Tönen, die durch die Reibung in der Luft auf ihrem Wege mehr verzehrt werden als die tiefen. „Bei den Tönen der gewöhnlichen musikalischen Scala ist allerdings", wie Helmboltz sagt, ,jene Abnahme äusserst unbedeutend, bei sehr hohen kann sie aber sehr merklich werden. Wenn man berechnet, wie weit sich ein Zug ebener Schallwellen fortpflanzen muss, ehe seine Intensität durch Reibung auf die Hälfte vermindert wird, so findet man

\begin{tabular}{c|c|c}
\hline Ton & $\begin{array}{c}\text { Schwingungs- } \\
\text { zahl }\end{array}$ & $\begin{array}{c}\text { Weglänge } \\
\text { in m }\end{array}$ \\
\hline$a_{1}$ & 440 & 383545 \\
$a_{5}$ & 7040 & 1498 \\
$d_{8}$ & 38016 & 52,7 \\
$a_{\mathbf{1 1}}$ & 450560 & 0,365
\end{tabular}

Höhere Töne, auch wenn sie sich hervorbringen liessen, würden unfähig sein, sich durch längere Luftstrecken fortzupflanzen."

Inwieweit bei unseren Versuchen diese Benachtheiligung der höheren Töne bei Luftleitung, beziehungsweise Passirung von Widerständen sich geltend gemacht hat, das kann ich natürlich nicht sagen. Wohl aber möchte ich behaupten, dass sie vorbanden war; denn wenn bei meinen Versuchen einerseits eine deutliche Abschwächung schon bei Klängen begann, die viel tiefer als das Stimmgabel- $a$ lagen, so ist andererseits nicht zu vergessen, dass die von mir angewendeten Widerstände meist viel grösser waren als die freie Luft.

Vielleicht könnte man mit empfindlichen Apparaten die von dem betreffenden Widerstand absorbirten Energiemengen messen, indem man die Temperatur der Widerstände feststellt, je nachdem ein tiefer oder ein hoher Ton von gleicher objectiver Stärke sie durchsetzt. Bei hohen Tönen müssten sie sich mehr erwärmen.

Beachtenswerth scheint mir ausserdem noch die Thatsache, dass ich ebenso wie Wien für Klänge von 1000 bis etwa 3000 Schwingungen das Ohr nahezu gleich empfindlich fand. Diese Klänge blieben auch nach Passirung des Widerstandes gleich laut, wie sie es ja auch vor Passirung desselben waren.

1) H. Helmholtz, Ueber den Einfluss der Reibung in der Luft anf die Schallbewegung. Wissenschaftl. Abhandl. Bd. 1 S. 383, 1882 aus Verhandl. des naturhist. med. Vereins zu Heidelberg Bd. 3 S. 16. 1863. 
Die mir noch zum Schlusse übrigbleibende Frage, warum das $A$ sich in Lautheit und Vocalcharakter bei Weitem am besten erhält, weniger das $O, E, I$ und am allerwenigsten das $U$, kann ich leider nicht in völlig befriedigender Weise beantworten. Wahrscheinlich ist es jedoch, worauf mich Herr Prof. v. Grützner aufmerksam machte, dass das $A$ eben einen sehr starken und nicht allzu hohen Formanten besitzt, wofür sowohl die bekannten Curven von Hensen und Hermann sprechen, als auch die oben beschriebenen, im $\mathrm{K}$ ö ni g'schen Spiegel erhaltenen Bilder, bei denen nach Einschaltung von Widerständen die charakteristischen kleinen Zacken der $A$-Curven nur in ganz geringem Maasse abgeschwächt erschienen.

Zum Schlusse habe ich die Pflicht, meinem hochverehrten Lehrer, Herru Prof. Dr. P. v. Grützner, der mir die Anregung zu diesen Versuchen gab und mir bei Ausführung derselben stets in liebenswürdigster Weise mit Rath und That zur Seite stand, meinen grössten Dank auszusprechen. Zugleich danke ich allen Denen, die mich bei den oft langwierigen und abspannenden Versuchen mit Ohr und Stimme unterstützt haben. 